\title{
A Novel Adaptive Algorithm Addresses Potential Problems of Blind Algorithm
}

\author{
Muhammad Yasin and Muhammad Junaid Hussain \\ National University of Sciences and Technology, H-12, Islamabad, Pakistan \\ Correspondence should be addressed to Muhammad Yasin; myasinkhattak@gmail.com
}

Received 1 February 2016; Revised 17 April 2016; Accepted 3 May 2016

Academic Editor: Yu Jian Cheng

Copyright ( 2016 M. Yasin and M. J. Hussain. This is an open access article distributed under the Creative Commons Attribution License, which permits unrestricted use, distribution, and reproduction in any medium, provided the original work is properly cited.

\begin{abstract}
A hybrid algorithm called constant modulus least mean square (CMLMS) algorithm is proposed in order to address the potential problems existing with constant modulus algorithm (CMA) about its convergence. It is a two-stage adaptive filtering algorithm and based on least mean square (LMS) algorithm followed by CMA. A hybrid algorithm is theoretically developed and the same is verified through MatLab Software. Theoretical model is verified through simulation and its performance is evaluated in smart antenna in presence of a cochannel interfering signal and additive white Gaussian noise (AWGN) of zero mean. This is also tested in Rayleigh fading channel using digital modulation technique for Bit Error Rate (BER). Finally, a few computer simulations are presented in order to substantiate the theoretical findings with respect to proposed model. Corresponding results obtained with the use of only CMA and LMS algorithms are also presented for further comparison.
\end{abstract}

\section{Introduction}

Smart antenna is an active area of research. Most of the antenna manufactures and digital signal processing developers are in opinion that smart antenna provides a sole solution for signal quality and capacity improvement either to direct a beam towards a desired user or to minimize a mean squared error (MSE) to fulfill today requirements. In this regard, various researchers are striving to excel in these prospects. In [1], LLMS algorithm is developed using LMS-LMS algorithms for stable convergence to get optimum results, whereas the same author has proposed another algorithm for beamforming known as RLMS $[2,3]$ using a combined RLS-LMS algorithm to provide a robust performance. Similarly in [4], Feng et al. proposed a fast recursive total least squares (TLS) algorithm for adaptive FIR filtering, whereas in [5] a simple variable step size LMS (VSSLMS) adaptive algorithm is presented for simple, robust, efficient, fast convergence and low steady state MSE. In [6], live model of Bessel beamformer is developed along with its convergence analysis [7], whereas the modified Bessel beamformer is presented in $[8,9]$ with automatic gain control (AGC) for beamforming to avoid the operator involvement for adjusting the step size parameter that controls the convergence rate of the algorithm. In [10], generalized Sato algorithm (GSA) and the multimodulus algorithm (MMA) are modified by adding constellation information in their cost functions to improve equalizer performance, that is, the dynamic convergence process. In [11], a variable step size least mean fourth (LMF) algorithm is proposed that achieves a better performance than the LMF algorithm in different noise environments and is shown to achieve a lower steady state error than the traditional LMF algorithm. Dominique Godard [12] was the first to introduce a family of blind equalization algorithm like CMA that has potential problems about its convergence [13]. First problem is that its convergence is not guaranteed because the cost function/MSE is not convex and may have false minima. Second potential problem is that if there is more than one strong signal, the algorithm may acquire an undesired signal. This problem can be overcome if additional information about the desired signal is available [13], whereas practical application of the CMA has demonstrated a number of circumstances in which it fails to converge, or, equally bad from a practical standpoint, converges to a solution 


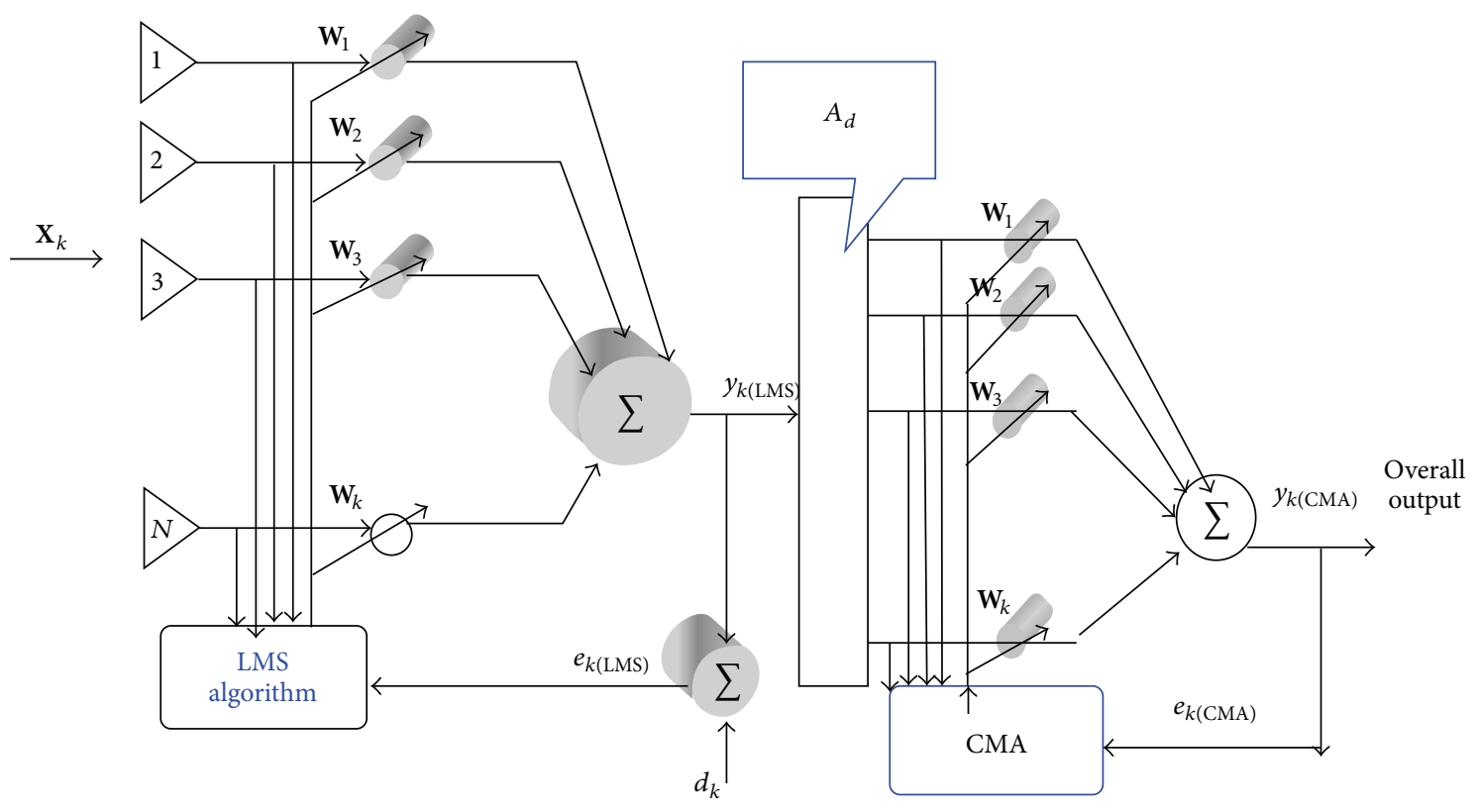

Figure 1: Proposed model.

which fails to equalize the input signal [14]. Those who have proposed various forms of CMA include supervised CMA (SCMA) [15], normalized CMA (N-CMA) [15] and modified CMA (M-CMA) [16] to redress the convergence problems of CMA.

In this paper, the effectiveness of a hybrid algorithm called CMLMS algorithm is proposed in order to evade the use of potential problems existing with CMA algorithm regarding its convergence [13-19]. Its performance is evaluated in an adaptive linear array having multiple inputs including the presence of a cochannel interfering signal, AWGN of zero mean and Rayleigh fading channel. Finally, a few computer simulations are presented in order to validate the theoretical findings with respect to the proposed model.

The paper is planned as follows. Section 2 explains the proposed model for the adaptive antenna array system. Section 3 presents mathematical model of the CMLMS algorithm. Computer simulations are provided in Section 4. Results are presented in Section 5. Section 6 concludes the paper.

\section{Proposed Model}

In this section, we model the adaptive antenna array system with a hybrid algorithm that consists of LMS algorithm followed by CMA as shown in Figure 1. We explain the said proposed model that involves the use of LMS (nonblind) and CMA (blind) algorithms. Both of them are alienated by an array image factor. LMS is a nonblind algorithm that requires a reference signal, also known as training signal, to update its complex weight vector. During the training period, the training signal is sent by the transmitter to the receiver and receiver uses this information to compute new weight for convergence to form a beam in the desired direction, whereas CMA is a blind algorithm that does not require a reference signal to train the adaptive weights but beamformer output is used as feedback to train the beamformer for optimum convergence, based on the idea of reducing system overhead and maintaining gain on the signal while minimizing the total output energy. As a result, a number of bits for transmitting information are increased which leads to enhancing capacity.

This arrangement as shown in Figure 1 is made in order to address potential problems existing with CMA algorithm with respect to its convergence.

Section 1 of the hybrid algorithm produces an output $y_{k \text { (LMS) }}$ that is calculated by using (1) and updates its weights using (6). This output $y_{k \text { (LMS) }}$ is estimated by LMS algorithm which is then fed into Section 2 (i.e., CMA) after it has been multiplied by the image of the desired signal array factor. It is pertinent to mention that error signal used for adjustment of adaptive system by optimizing the weight vector of LMS algorithm is sourced from external reference signal $\left(d_{k}\right)$ that is estimated by using (7), whereas, for updating the CMA weights, reference signal is obtained from its self-referenced version (i.e., output of CMA is used as feedback to train the CMA for optimum convergence) and this is estimated by using (11).

Thus, in the proposed scheme as shown in Figure 1, the immediate output $y_{k \text { (LMS) }}$ yielded from Section 1 is multiplied by image of the desired signal array factor $\left(A_{d}\right)$ that results in a filtered signal $\left(A_{d} y_{k(\mathrm{LMS})}\right)$. This filtered signal is further processed by CMA section using (8) and, eventually, we get an optimum output using (14) through adaptation process using (15) by proposed beamformer with input signal array vector $\mathbf{X}_{k}$.

The number and types of parameters within proposed model depend on the computational structure chosen for the smart antenna system. However, this structure may be reduced to general adaptive filter structure that has been proven useful for adaptive filtering tasks as shown in Figure 2. 


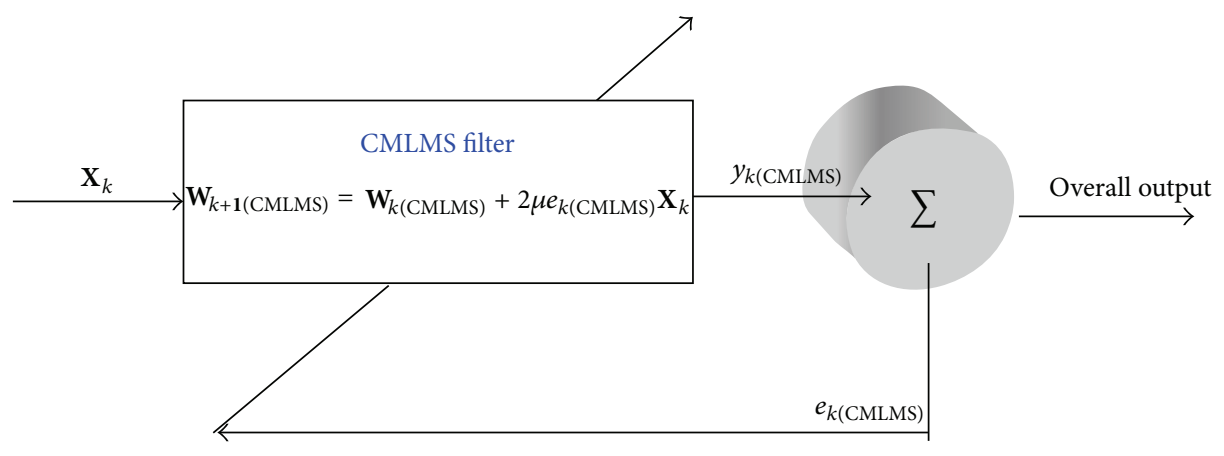

FiguRE 2: CMLMS adaptive filter structure.

\section{Mathematical Model}

Considering a linear beamformer having a hybrid algorithm that combines the use of LMS algorithm followed by CMA in an arrangement using multiple inputs at its array's elements as shown in Figure 1 then output of the LMS section at $k$ th iteration can be defined as

$$
y_{k(\mathrm{LMS})}=\mathbf{W}_{\mathrm{LMS}} \mathbf{X}_{k}
$$

where $k$ is the iteration number.

The signal array vector received on the elements of antenna is written by

$$
\mathbf{X}_{k}=\left[x_{1}, x_{2}, \ldots, x_{N}\right]^{T},
$$

where $T$ signifies the transpose of the vector within the brackets and linear array having $\mathrm{N}$-element composed of isotropic radiating antenna elements.

As signal array vector consists of desired and other interfering signals $[20,21]$; therefore, it can also be written as

$$
\mathbf{X}_{k}=s_{d}(k) a\left(\theta_{d}\right)+\sum_{i=1}^{L} s_{i}(k) a\left(\theta_{i}\right)+n(k)
$$

where $s_{d}$ and $s_{i}$ are the desired and interfering signals arriving at the array at angles $\theta_{d}$ and $\theta_{i}$, respectively. $L$ is the number of interfering signals and $n$ is a white and zero mean complex Gaussian noise at the array elements. $a\left(\theta_{d}\right)$ and $a\left(\theta_{i}\right)$ are the steering vectors for the desired and interfering signals, respectively, which is also known as image of the desired and interfering signals array factor. However, when LMS algorithm converges, its output tends to approach desired signal $\left(s_{d}\right)$ with both interfering signal $\left(s_{i}\right)$ and Gaussian noise $(n)$ being suppressed. Therefore, steering vector or image of the desired signal array factor $\left(A_{d}\right)$ is described as

$$
A_{d}(\theta)=a\left(\theta_{d}\right)=\left[1, e^{-j \phi}, \ldots, e^{-j(N-1) \phi}\right],
$$

where $\phi=(2 \pi d / \lambda) \sin \theta$ is the phase shift observed at each sensor due to the angle of arrival of the wavefront and assume $d$ is the uniform distance between array elements. $\lambda=c / f$ where $f$ is in Hertz. Therefore, the steering vector or image of the desired signal array factor $\left(A_{d}\right)$ can be written as

$$
\begin{aligned}
A_{d}(\theta) & =a\left(\theta_{d}\right) \\
& =\left[1, e^{-j(2 \pi / \lambda) d \sin (\theta)}, \ldots, e^{-j(2 \pi / \lambda) d(N-1) \sin (\theta)}\right] .
\end{aligned}
$$

The input stage of the CMLMS scheme is based on the LMS algorithm as shown in arrangement in Figure 1 with its weight vector at $(k+1)$ th iteration updated accordingly and is given by

$$
\mathbf{W}_{k+\mathbf{1}(\mathrm{LMS})}=\mathbf{W}_{k(\mathrm{LMS})}+2 \mu e_{k(\mathrm{LMS})} \mathbf{X}_{k},
$$

where $\mu$ is the step size and its error signal used for adjustment of adaptive system by optimizing the weight vector is given by

$$
e_{k(\mathrm{LMS})}=d_{k}-y_{k(\mathrm{LMS})} \text {, }
$$

where $d_{k}$ is the reference signal, also known as pilot signal. This reference signal is used as desired response from the adaptive processor connected with the antenna array elements which guide the beamformer to map the main beam towards a specified direction only. Output of the LMS section is denoted by $y_{k \text { (LMS) }}$ at $k$ th iteration as defined in (1). With this filtered signal (output of the LMS section) forming the input to the following CMA section, the input signal vector of the CMA section becomes

$$
\mathbf{X}_{k(\mathrm{CMA})}=A_{d} y_{k(\mathrm{LMS})},
$$

where $A_{d}$ is the image array factor of the desired signal.

Putting value of (1) into (8) then

$$
\mathbf{X}_{k(\mathrm{CMA})}=A_{d} \mathbf{W}_{\mathrm{LMS}} \mathbf{X}_{k} .
$$

For the CMA stage, its weight vector is updated according to

$$
\mathbf{W}_{k+\mathbf{1}(\mathrm{CMA})}=\mathbf{W}_{k(\mathrm{CMA})}+2 \mu e_{k(\mathrm{CMA})} \mathbf{X}_{k(\mathrm{CMA})},
$$

where $e_{k(\mathrm{CMA})}$ is the error signal and is given by

$$
e_{k(\mathrm{CMA})}=\left(y_{k(\mathrm{CMA})}-\frac{y_{k(\mathrm{CMA})}}{\left|y_{k(\mathrm{CMA})}\right|}\right),
$$


where $y_{k(\mathrm{CMA})}$ is the output of the CMA section and is given by

$$
y_{k(\mathrm{CMA})}=\mathbf{W}_{\mathrm{CMA}} \mathbf{X}_{k(\mathrm{CMA})} .
$$

Putting value of (9) into (12) then

$$
y_{k(\mathrm{CMA})}=\mathbf{W}_{\mathrm{CMA}} A_{d} \mathbf{W}_{\mathrm{LMS}} \mathbf{X}_{k} \text {. }
$$

Equation (13) finally becomes the output of the CMLMS beamformer and is given by

$$
y_{k(\mathrm{CMLMS})}=\mathbf{W}_{\mathrm{CMLMS}} \mathbf{X}_{k} \text {, }
$$

where $\mathbf{W}_{\mathrm{CMLMS}}$ is the required optimum solution or optimal weight vector for proposed beamformer with input signal array vector $\mathbf{X}_{k}$ and is given by

$$
\mathbf{W}_{k+\mathbf{1}(\mathrm{CMLMS})}=\mathbf{W}_{k(\mathrm{CMLMS})}+2 \mu e_{k(\mathrm{CMLMS})} \mathbf{X}_{k} \text {, }
$$

where $e_{k \text { (CMLMS) }}$ is overall error signal and is given by

$$
e_{k(\mathrm{CMLMS})}=\left(y_{k(\mathrm{CMLMS})}-\frac{y_{k(\mathrm{CMLMS})}}{\left|y_{k(\mathrm{CMLMS})}\right|}\right)
$$

and $\mu$ is the step size which is given by [22]

$$
0<\mu<\frac{1}{\lambda_{\max }},
$$

where $\lambda_{\max }$ is the largest eigenvalue of autocorrelation matrix which is denoted by $\mathbf{R}$. This autocorrelation matrix describes correlation between various elements of signal array vector $\mathbf{X}_{k}$. The stability of proposed algorithm is maintained by choosing the step size parameter by trial-and-error method within specified range as stated above in (17).

Equation (15) implies that the adaptive process will finally converge to mean square error, as the adaptation progresses. In summary, the proposed beamformer performs the following steps.

Step 1. Obtain $\mathbf{X}_{k}$ in (2) by signal array vector.

Step 2. Get output $\left\{y_{k \text { (CMLMS) }}\right\}$ of the CMLMS beamformer in (14) concluding both parts of LMS and CMA.

Step 3. Calculate overall error signal $\left\{e_{k \text { (CMLMS) }}\right\}$ for optimizing the weight vector in (16).

Step 4. Calculate the robust adaptive beamformer weights $\left\{\mathbf{W}_{k+\mathbf{1}(\mathrm{CMLMS})}\right\}$ in (15).

Step 5. Repeat the above steps in closed loop to get optimum results.

\section{Simulation Results}

In this section, performance analysis is carried out for proposed algorithm and the same is also compared with LMS and CMA algorithms for further assessment. We are adapting a strategy that an incoming signal coming in at a certain

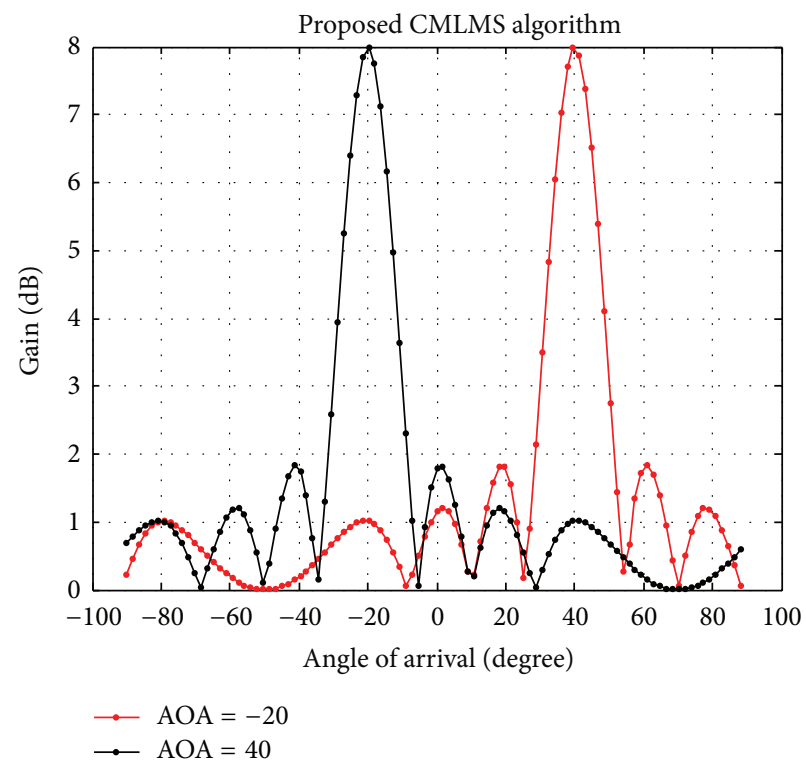

FIGURE 3: Beam pattern achieved by CMLMS algorithm.

angle (here $40^{\circ} \&-20^{\circ}$ ) using $N$ (here $N=8$ ) antennas and suppressing all other incoming signals at the same time. This assumption is met while discussing our results.

We consider the following parameters for the simulations purpose:

(i) A linear array model with 8 elements and spacing between two elements is $0.5 \lambda$.

(ii) The channels are AWGN and Rayleigh fading with interference signal included.

(iii) All weight vectors for algorithms under analysis are initially set at zero.

(iv) Number of samples is fifty for all simulations.

AOA for desired signals are set at -20 and 40 degrees as shown in Figure 3 for proposed algorithm and subsequent MSE is obtained. Optimum gain towards desired users with minimum sidelobe level (SLL) is obtained. Small SLL indicates that proposed algorithm will get less interference that will enhance security and provide quality signal to desired users.

Substantial reduction in MSE is also observed which eliminates the potential problems existing with CMA regarding its convergence. Convergence is the process of minimizing the power of the error signal. The performance curve as shown in Figure 4 indicates that CMLMS has minimum MSE and starts to converge from the iteration number 30 when measured after 50 iterations.

In this case, step size is set at 0.0001 by trial-and-error method but within range as specified in (17). It is to be noted that step size has significant effect on convergence and stability of the proposed beamformer. The step size within bounded range gives marked improvement in reduction of SLL and in error minimization. Obtained results are summarized in Table 1. 
TABLE 1: Input and output estimates.

\begin{tabular}{lcccc}
\hline & $\begin{array}{c}\text { Input parameter } \\
\text { Number of elements }\end{array}$ & Element spacing & Output parameter \\
AOA (degree) & 8 & $0.5 \lambda$ & 1.818 & Gain $(\mathrm{dB})$ \\
\hline $40^{\circ} \&-20^{\circ}$ & $8 \mathrm{~dB})$ & 8.001 \\
\hline
\end{tabular}

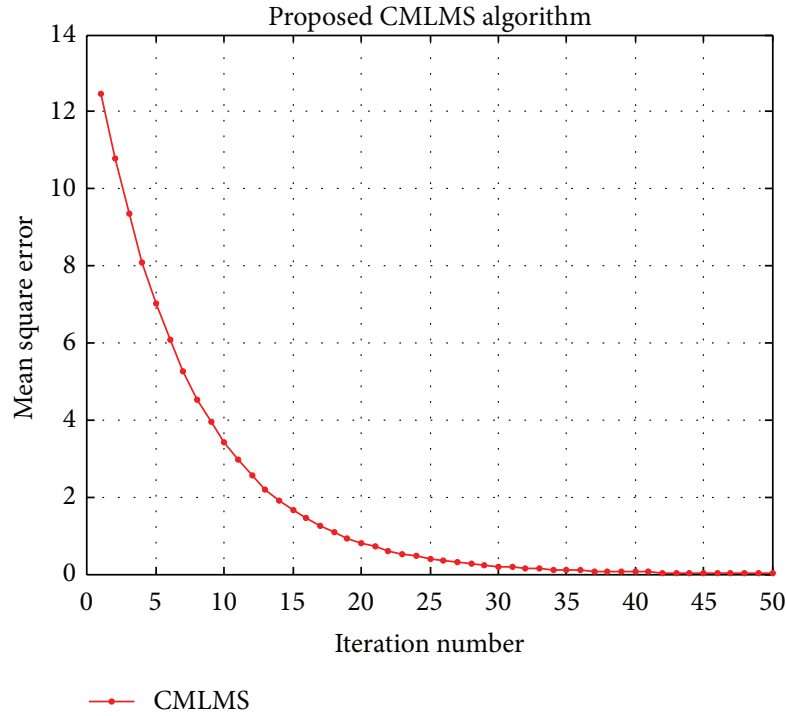

FIGURE 4: Convergence behavior of CMLMS algorithm.

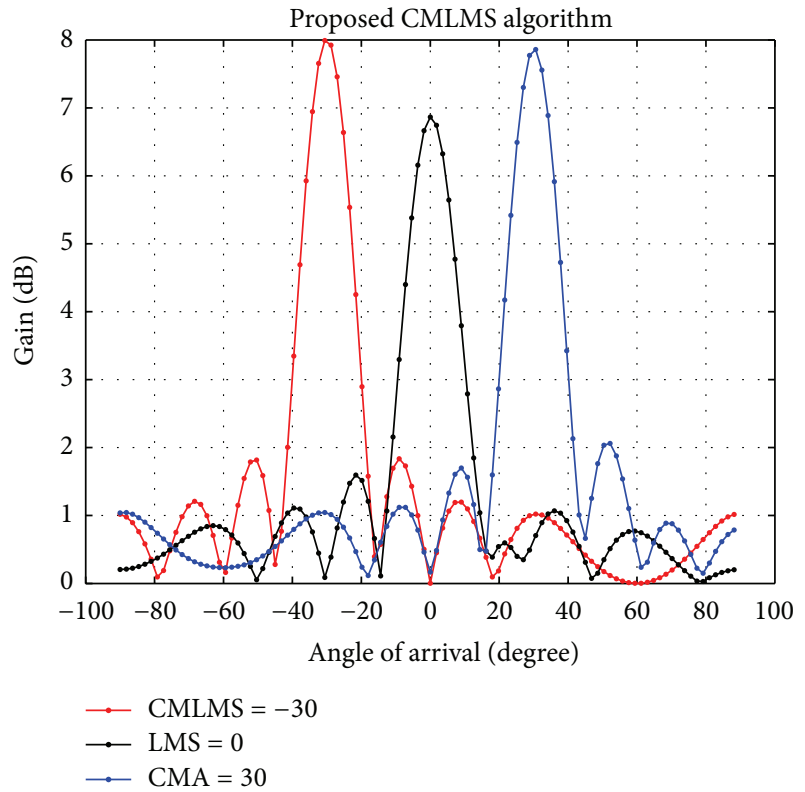

FIGURE 5: Beam patterns achieved by CMLMS, LMS, and CMA algorithms.

\section{Performance Comparison}

5.1. Performance with respect to Array Gain. Proposed algorithm is also compared with CMA and LMS algorithms as shown in Figure 5 for its improved performance in terms of array gain with different AOA set for desired users.

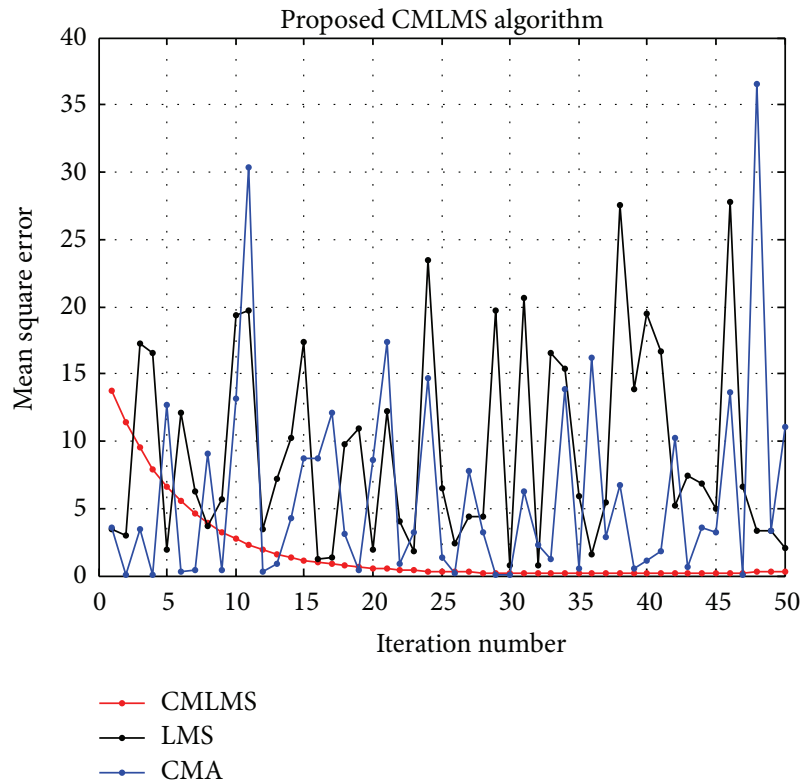

Figure 6: Convergence behaviors of CMLMS, LMS, and CMA algorithms.

TABLE 2: Performance analysis.

\begin{tabular}{lccc}
\hline \multirow{2}{*}{ Algorithms } & $\begin{array}{c}\text { Input parameter } \\
\text { AOA (degree) }\end{array}$ & \multicolumn{2}{c}{ Output parameter } \\
& $30^{\circ}$ & 2.034 & Gain $(\mathrm{dB})$ \\
\hline CMA & $0^{\circ}$ & 1.596 & 6.859 \\
LMS & $-30^{\circ}$ & 1.818 & 8.001 \\
CMLMS &
\end{tabular}

You see the optimum array gain with respect to CMA and LMS algorithms. The SLL of proposed algorithm is slightly less/greater than CMA and LMS algorithms, respectively, but with constant amplitude. It means that CMLMS algorithm saves power by reduction in SLL as compared to CMA. However, at the same time, it has more array gain in comparison with LMS with slightly large SLL but this can be compromised keeping large gain and robustness in MSE as shown in Figure 6.

Because of this, it may be more useful where signal statistics vary rapidly with time. In this case, step size is set at 0.0008 by trial-and-error method for algorithms under consideration and all other parameters remain the same as described above for better comparison. The performance analysis of study algorithms are summarized in Table 2.

5.2. Performance with respect to Convergence. If we compare and observe the MSE curves as shown in Figure 6, then we conclude that CMLMS algorithm has minimum MSE and 


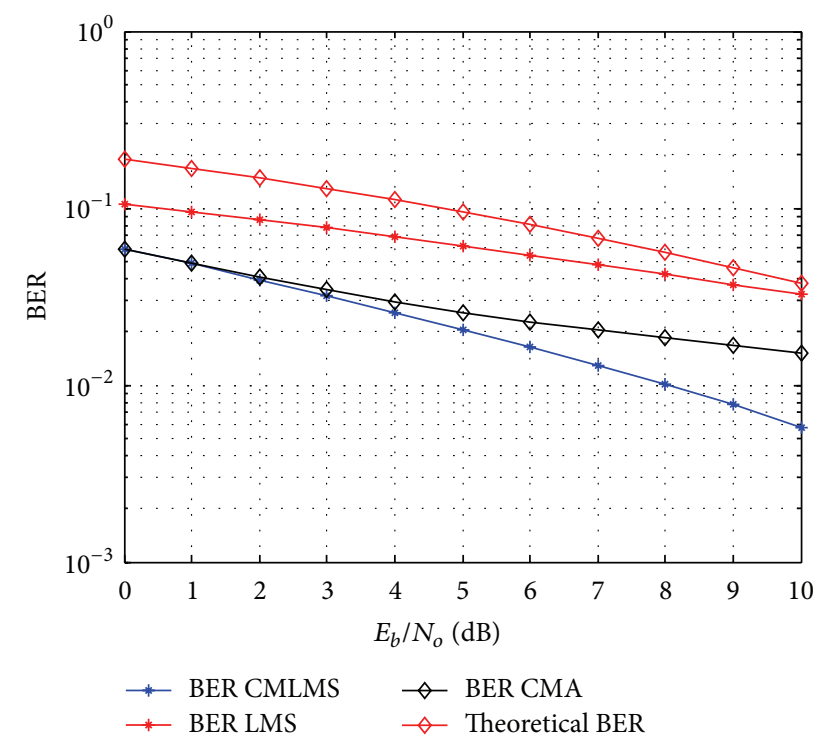

FIgURE 7: BER performance of CMLMS, LMS, and CMA algorithms.

follows steady path. The learning curves of the understudy algorithms exhibit that CMA and LMS algorithms have fluctuation and their performance is worst than the performance of the proposed scheme.

It means that the proposed algorithm illustrates clear performance advantages over other CMA and LMS techniques simulated for comparisons in both areas of convergence speeds and MSE floor. Therefore, proposed algorithm can achieve lower MSE and faster robust convergence than CMA and typically LMS algorithm for the same adaptation size or iterations which is extremely important in the application of wireless cellular communication where signal statistics vary rapidly with time.

5.3. Performance with respect to Bit Error Rate. Proposed algorithm is also operated in digital domain in order to assess its performance and compared with CMA and LMS algorithms as shown in Figure 7. All these algorithms are tested in Rayleigh fading channel using digital modulation technique to measure their Bit Error Rates (BERs). The simulations are designed for quadrature amplitude modulation (QAM) signal with 0 to $10 \mathrm{~dB}$ SNR and step size is fixed at $8.5616 e-$ 04 . Values for BERs are obtained through the semianalytic technique and the same values are also compared with the theoretical BERs at different SNR.

The BER performance curves are shown in Figure 7 which indicates that the computed values of BER are smaller than theoretical values of BER; means BER performance is greatly improved. It is realized that the CMLMS is the best in performance followed by CMA and LMS algorithms. Figure 7 shows the BER performance of our proposed scheme and achieves a BER of $10^{-2}$ at an SNR of $8 \mathrm{~dB}$. The data obtained from Figure 7 is provided in Table 3 which indicates that proposed scheme outperforms both CMA and LMS algorithms.
TABLE 3: BER performance analysis.

\begin{tabular}{lcccc}
\hline$E_{b} / N_{o}(\mathrm{~dB})$ & BER theory & BER LMS & BER CMA & BER CMLMS \\
\hline 0 & 0.1889 & 0.1041 & 0.0589 & 0.0586 \\
1 & 0.1682 & 0.0949 & 0.0492 & 0.0483 \\
2 & 0.1482 & 0.0859 & 0.0410 & 0.0394 \\
3 & 0.1292 & 0.0772 & 0.0343 & 0.0318 \\
4 & 0.1114 & 0.0690 & 0.0292 & 0.0255 \\
5 & 0.0951 & 0.0613 & 0.0253 & 0.0204 \\
6 & 0.0804 & 0.0542 & 0.0225 & 0.0163 \\
7 & 0.0673 & 0.0478 & 0.0204 & 0.0129 \\
8 & 0.0559 & 0.0421 & 0.0185 & 0.0101 \\
9 & 0.0461 & 0.0371 & 0.0168 & 0.0077 \\
10 & 0.0378 & 0.0328 & 0.0151 & 0.0057 \\
\hline
\end{tabular}

If we take one computed BER value of LMS, CMA, and CMLMS algorithms, say at $5 \mathrm{~dB}$ SNR with respect to BER reduction capabilities, then we have computed $B E R$ value of LMS, CMA, and CMLMS algorithms at $5 \mathrm{~dB}$ SNR which are $0.0613,0.0253$, and 0.0204 , respectively.

The BER values of CMLMS are $33.27 \%$ (0.0204 is 33.27\% of 0.0613 ) as compared to LMS algorithm whereas the BER values of CMLMS are $80.63 \%$ (0.0204 is $80.63 \%$ of 0.0253 ) as compared to CMA. Then BER reduction capability of CMLMS at $5 \mathrm{~dB}$ SNR is $66.73 \%$ as compared to LMS algorithm whereas reduction of the BER for CMLMS is $19.37 \%$ compared to CMA. Therefore, CMLMS algorithm is more cost effective for wireless cellular communication system as compared to LMS and CMA algorithms in this respect.

\section{Discussion on Results}

The following outcomes are deducted from the given results:

(i) The proposed algorithm does not always require an external reference signal for its operation but adapts itself entirely through self-referencing (i.e., output of proposed algorithm is used as feedback to train the beamformer for its optimum convergence) to the desired signal using the correct reference signal during the initial a few iterations only for LMS. The configuration (as shown in Figure 1) uses a (nonblind) LMS trained equalizer first to open the intersymbol interference (ISI) communication eye and when the eye was open, the training finished/LMS switched out and the system reverted to blind decision feedback.

(ii) The convergence of proposed algorithm is robust in presence of noise and follows steady path. Therefore, proposed algorithm may correctly address the potential problems existing with CMA algorithm regarding its convergence.

(iii) The steady state MSE of proposed algorithm is found to be the most favorable as compared to CMA and LMS. An adaptive system with small minimum MSE indicates that this system has accurately modeled, predicted, adapted, and/or converged to a solution for the given system. 
(iv) The proposed algorithm has achieved similar beam patterns as obtained with CMA and LMS but with optimum array gain as tabulated in Table 1 and shown in Figures 3 and 5.

(v) With optimum array gain, proposed algorithm enhances range.

(vi) The proposed algorithm directs its energy towards desired users only; therefore, it saves energy, due to which battery life installed at Base Transceiver Station (BTS) increases.

(vii) As there is no leakage of energy towards interferers, therefore minimum BTS is required to cover the service area and infrastructures cost may be reduced.

(viii) With optimum array gain and no leakage of energy towards interferers, security of subscribers may be enhanced.

(ix) Leakage of classified information/tapping may be reduced/restricted.

(x) Complexity of proposed algorithm is slightly more as compared to CMA and LMS when treated as single entity. However, at the same time, it has more array gain and steady state MSE in comparison with CMA and LMS as shown in Figure 6. Further, the proposed algorithm illustrates a clear performance advantages over other CMA and LMS techniques simulated for comparisons in both areas of convergence speeds and MSE floor. Because of this, it may be more useful where signal statistics vary rapidly with time. Therefore, its application is extremely important in wireless cellular communication where signal statistics vary rapidly.

(xi) Further, as powerful low cost digital signal processors (DSPs) are commercially available nowadays, therefore algorithm complexity or computational cost with respect to execution time would not make much difference. Besides, all other requirements are met by the proposed technique. So it is better to use proposed technique for getting aforesaid advantages for smart antenna.

(xii) BER reduction capability of CMLMS, say at $5 \mathrm{~dB}$ SNR, is $66.73 \%$ as compared to LMS algorithm whereas reduction of the BER for CMLMS is $19.37 \%$ compared to CMA. Hence, CMLMS algorithm provides cost effective solution for wireless cellular communication system as compared to LMS and CMA algorithms in this respect. If SNR value increases then BER reduction capability of CMLMS algorithm also increases as tabulated in Table 3.

\section{Conclusions}

In this paper, we have introduced a new promising technique for adaptive beamforming called CMLMS algorithm, being a breakthrough design delivering high forward gain and unmatched interference rejection. The performance of CMLMS with CMA and LMS algorithms is discussed in the noise and Rayleigh fading channels models. Relevant results depict that smart antenna equipped with proposed algorithm can increase the number of active users (means increase in capacity) in $3 \mathrm{G}$ and beyond system significantly without losing of performance quality.

\section{Competing Interests}

The authors declare that they have no competing interests.

\section{References}

[1] J. A. Srar, K.-S. Chung, and A. Mansour, "Adaptive array beamforming using a combined LMS-LMS algorithm," IEEE Transactions on Antennas and Propagation, vol. 58, no. 11, pp. 3545-3557, 2010.

[2] J. A. Srar and K. S. Chung, "Adaptive array beamforming using a combined RLS-LMS algorithm," in Proceedings of the 14th AsiaPacific Conference on Communications, pp. 1-5, Tokyo, Japan, October 2008.

[3] J. A. Srar and K.-S. Chung, "Performance of RLMS algorithm in adaptive array beam forming," in Proceedings of the 11th IEEE Singapore International Conference on Communication Systems (ICCS '08), pp. 493-498, Guangzhou, China, November 2008.

[4] D. Feng, X. Zhang, D. Chang, and W. Zheng, "A fast recursive total least squares algorithm for adaptive FIR filtering," IEEE Transactions on Signal Processing, vol. 52, no. 10, pp. 2729-2737, 2004.

[5] T. I. Haweel, "A simple variable step size LMS adaptive algorithm," International Journal of Circuit Theory and Applications, vol. 32, no. 6, pp. 523-536, 2004.

[6] M. Yasin and P. Akhtar, "Design and performance analysis of live model of Bessel beamformer for adaptive array system," COMPEL-The International Journal for Computation and Mathematics in Electrical and Electronic Engineering, vol. 33, no. 4, pp. 1434-1447, 2014.

[7] M. Yasin and P. Akhtar, "Convergence analysis of Bessel beamformer and its comparison with LMS in adaptive array system," COMPEL - The International Journal for Computation and Mathematics in Electrical and Electronic Engineering, vol. 34, no. 3, pp. 952-961, 2015.

[8] M. Yasin and P. Akhtar, "Mathematical model of bessel beamformer with automatic gain control for smart antenna array system," Arabian Journal for Science and Engineering, vol. 39, no. 6, pp. 4837-4844, 2014.

[9] M. Yasin, P. Akhtar, and A. H. Pathan, "Mathematical model of bessel beamformer with automatic gain control for smart antenna array system in Rayleigh fading channel," IEEJ Transactions on Electrical and Electronic Engineering, vol. 9, no. 3, pp. 229-234, 2014.

[10] L. He and S. A. Kassam, "Convergence analysis of blind equalization algorithms using constellation-matching," IEEE Transactions on Communications, vol. 56, no. 11, pp. 1765-1768, 2008.

[11] S. M. Asad, A. Zerguine, and M. Moinuddin, "On the convergence analysis of a variable step-size LMF algorithm of the quotient form," in Proceedings of the IEEE International Conference on Acoustics, Speech, and Signal Processing (ICASSP '10), pp. 3722-3725, Dallas, Tex, USA, March 2010.

[12] F. B. Gross, Smart Antennas for Wireless Communications with MATLAB, McGraw-Hill, New York, NY, USA, 2005. 
[13] C. B. Dietrich Jr., Adaptive arrays and diversity antenna configurations for handheld wireless communication terminals [Ph.D. thesis], Virginia Polytechnic Institute, State University, 2000, chapter 3.

[14] J. R. Treichler, V. Wolff, and C. R. Johnson Jr., "Observed misconvergence in the constant modulus adaptive algorithm," in Proceedings of the 25th Asilomar Conference on Signals, Systems and Computers, pp. 663-667, Pacific Grove, Calif, USA, 1991.

[15] A. Özen, I. Kaya, and B. Soysal, "A supervised constant modulus algorithm for blind equalization," Wireless Personal Communications, vol. 62, no. 1, pp. 151-166, 2012.

[16] I. Chahed, J. Belzile, and A. B. Kouki, "Blind decision feedback equalizer based on high order MCMA," in Proceedings of the IEEE Canadian Conference on Electrical and Computer Engineering (CCECE '04), vol. 4, pp. 2111-2114, Niagara Falls, Canada, May 2004.

[17] D. L. Jones, "A normalized constant modulus algorithm," in Proceedings of the 29th IEEE Asilomar Conference on Signals, Systems, and Computers, vol. 1, pp. 694-697, Pacific Grove, Calif, USA, 1996.

[18] S. S. Moghaddam and M. S. Moghaddam, "A comprehensive survey on antenna array signal processing," Trends in Applied Sciences Research, vol. 6, no. 6, pp. 507-536, 2011.

[19] A.-J. van der Veen and A. Paulraj, "An analytical constant modulus algorithm," IEEE Transactions on Signal Processing, vol. 44, no. 5, pp. 1136-1155, 1996.

[20] L. Zhang, H. C. So, L. Ping, and G. Liao, "Effective beamformer for coherent signal reception," Electronics Letters, vol. 39, no. 13, pp. 949-951, 2003.

[21] L. Zhang, H. C. So, L. Ping, and G. Liao, "Adaptive multiplebeamformers for reception of coherent signals with known directions in the presence of uncorrelated interferences," Signal Processing, vol. 84, no. 10, pp. 1861-1873, 2004.

[22] B. Widrow and S. D. Stearns, Adaptive Signal Processing, Pearson Education, Upper Saddle River, NJ, USA, 1985. 


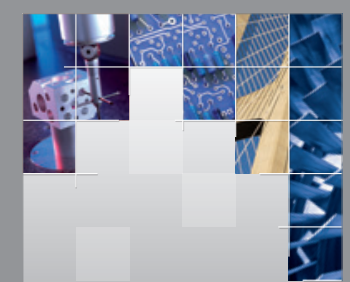

\section{Enfincering}
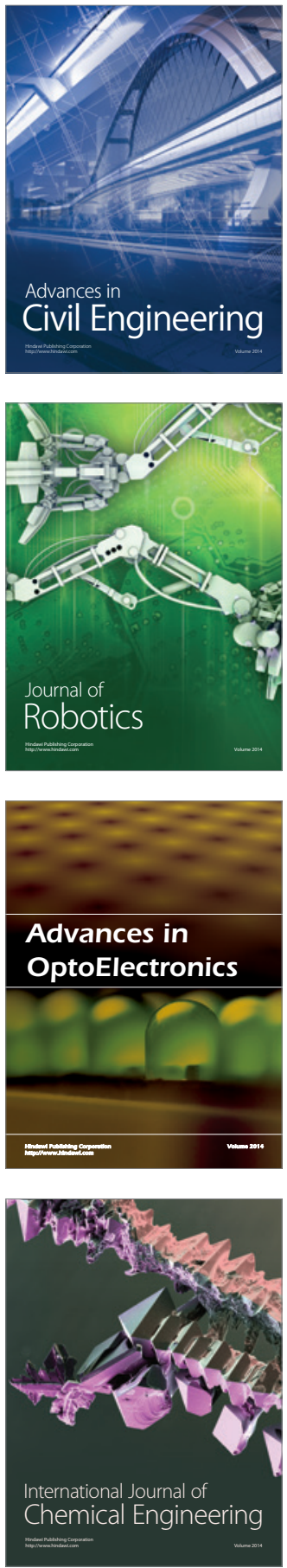

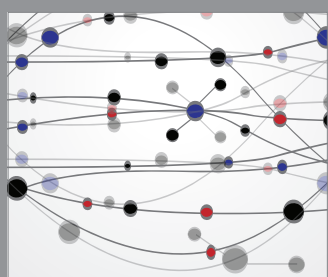

The Scientific World Journal

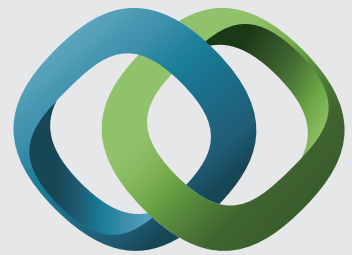

\section{Hindawi}

Submit your manuscripts at

http://www.hindawi.com
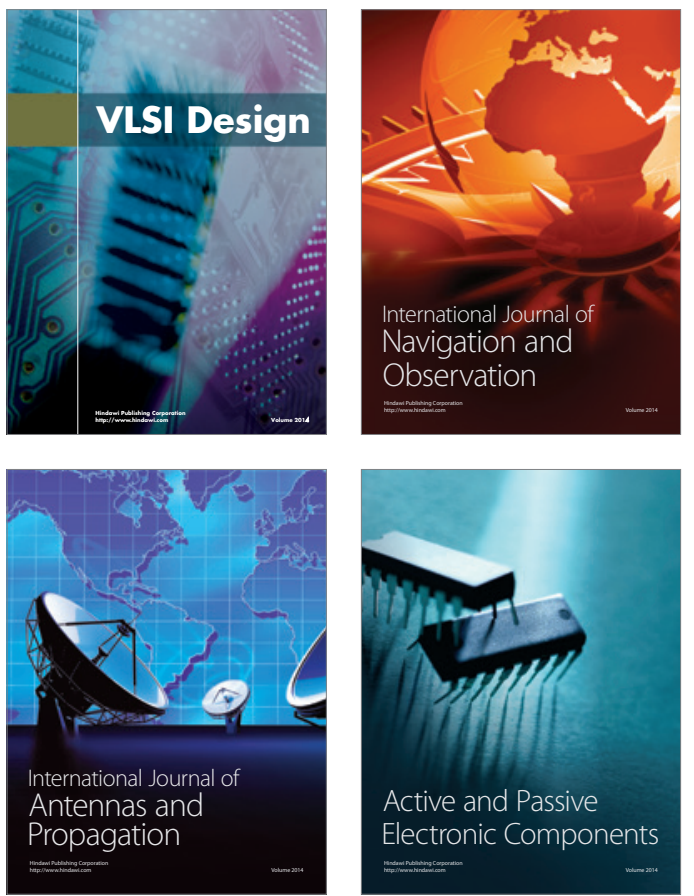
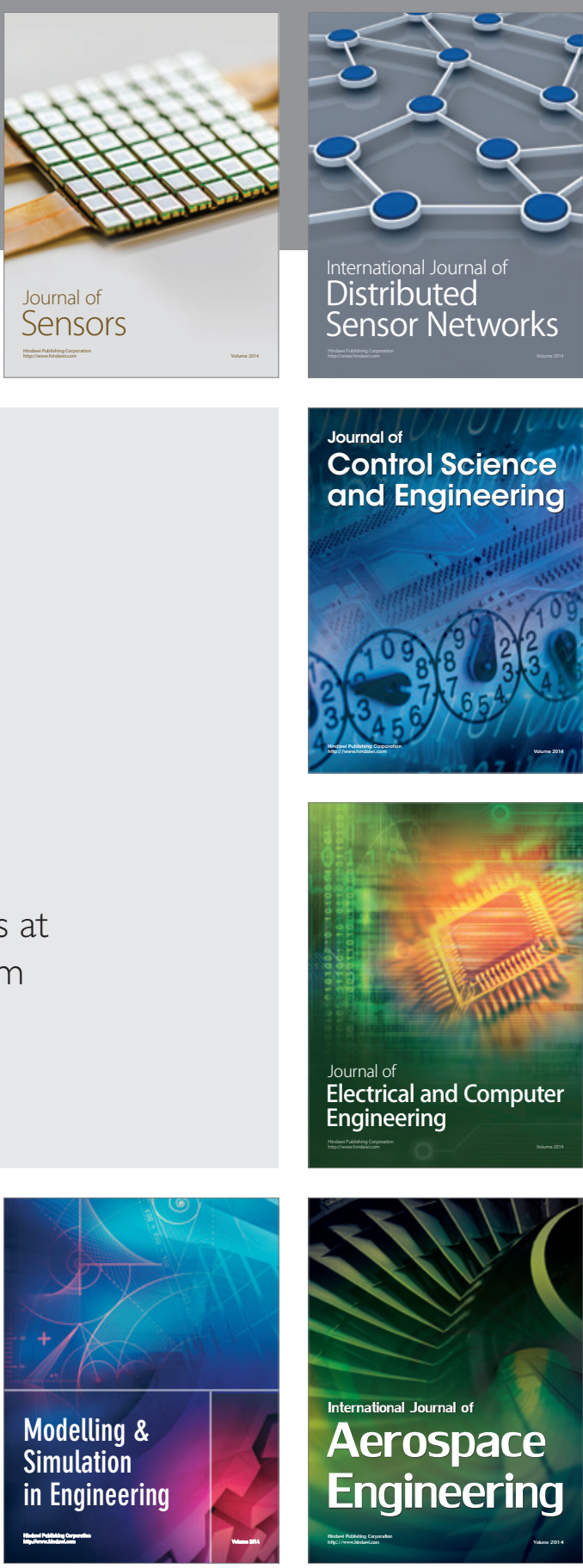

International Journal of

Distributed

Sensor Networks

Journal of

Control Science

and Engineering
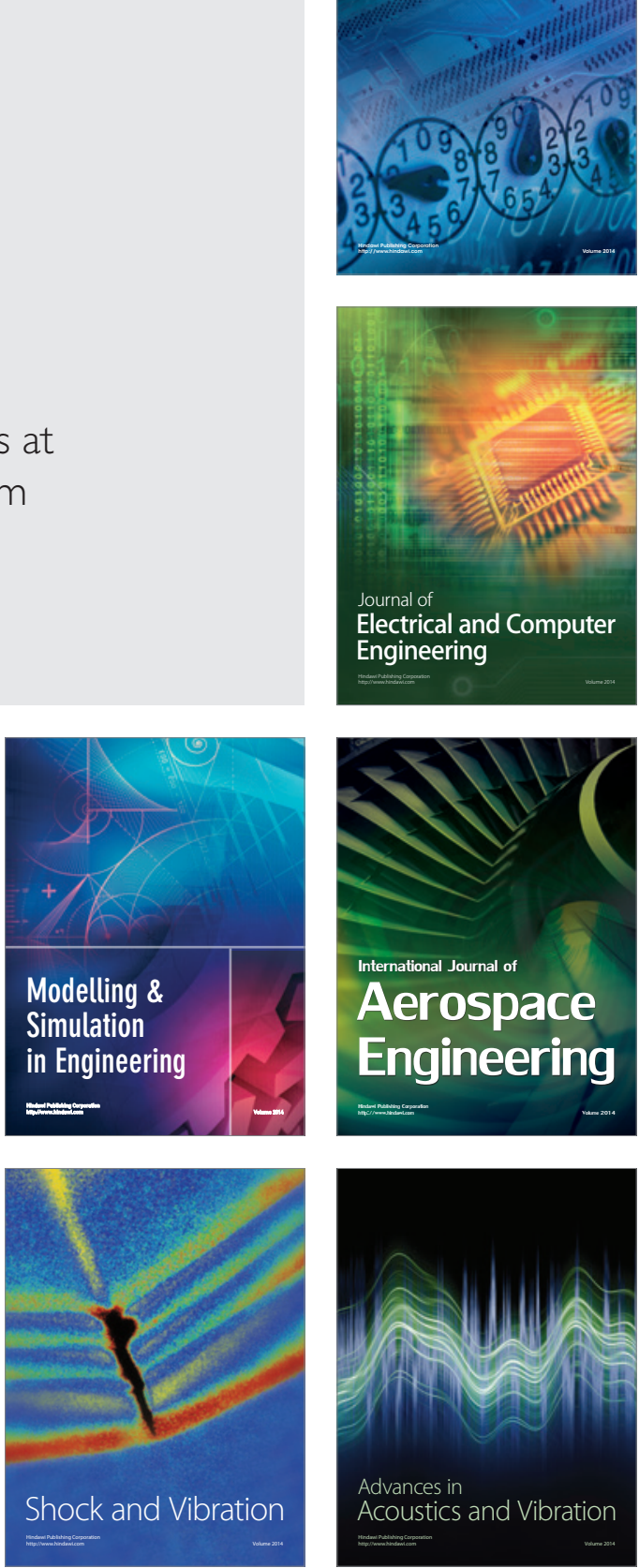